\title{
Karydakis Flap Reconstruction for Pilonidal Disease
}

\author{
Aybala Yildiz ${ }^{1}$, Alp Yildiz ${ }^{1 *}$, Veysel Baris Turhan², Engin Kucukdiler ${ }^{3}$ \\ and Erkan Karacan ${ }^{3}$ \\ ${ }^{1}$ Department of General Surgery, Yenimahalle Training and Research Hospital, Yildirim \\ Beyazit University, Ankara, Turkey \\ ${ }^{2}$ Department of General Surgery, Kecioren Training and Research Hospital, Ankara, \\ Turkey \\ ${ }^{3}$ Department of General Surgery, Aydin State Hospital, Aydin, Turkey \\ *Corresponding Author: Alp Yildiz, Department of General Surgery, Yenimahalle \\ Training and Research Hospital, Yildirim Beyazıt University, Ankara, Turkey.
}

Received: May 08, 2020

Published: June 22, 2020

(c) All rights are reserved by Alp Yildiz.,

et al.

\section{Abstract}

The reported incidence rate of pilonidal disease is 25 per 100,000 people. Initially, the pathogenesis was considered to be congenital. Today, however, the theory that it is acquired is more widely accepted. Work by Georgios Karydakis who highlighted 3 main factors contributing to pilonidal disease had a pivotal role in this paradigm shift. As the primary treatment still surgery, we present our karydakis flap experience in this study. Fourty-four patients has enrolled for this study. All patients has diagnosed pilonidal sinus disease and treated by the same surgical team with Karydakis flap reconstruction. The most common post-operative complication was fluid collection. 2 patients developed fluid collection under the flap site which reduce spontaneously. No hemorrhagia has occurred. 1 patient developed wound infection. No hematoma, bleeding occurred and no need for secondary powder application. No flap necrosis occurred, also wound dehiscence and early recurrence were not found. When low recurrence rates, patient comfort and cosmetic results are evaluated together, Karydakis technique emerges a method that is preferred by physicians and patients.

Keywords: Pilonidal Disease; Karydakis Flap; Complications

\section{Introduction}

The reported incidence rate of pilonidal disease (PD) is 25 per 100,000 people. Initially, the pathogenesis was considered to be congenital $[1,2]$. Today, however, the theory that it is acquired is more widely accepted. Work by Georgios Karydakis [1,3,4] who highlighted 3 main factors contributing to pilonidal disease (loose hair, an external force that facilitates insertion of hair into the skin and an underlying vulnerability of natal cleft skin), had a pivotal role in this paradigm shift. The first 2 factors are related to personal hygiene and lifestyle and their modulation can influence the initiation, development and recurrence of pilonidal disease [1-5]. Armstrong and Barcia [5] reported that improved hygiene, an active lifestyle and hair control in the natal cleft area decreased the need for surgical procedures and resulted in faster return to work. However, the third factor can be modified only surgically [1-5]. In this paper we present our experience of karydakis flap reconstruction on pilonidal sinus disease management.

\section{Patients and Methods}

Fourty-four patients included this study. All patients has diagnosed pilonidal sinus disease and treated by the same surgical team with Karydakis flap reconstruction. All patients underwent routine investigation before surgery. On premedication wide spectrum antibiotics has used 30 minutes before surgery.

All procedures performed under spinal anaesthesia in prone jack knife position. Methylene blue used to help visualisation of the sinus tracts. An elliptodi incision was made to excise the pilonidal sinus. The sharp ends of the ellipse has incised $3 \mathrm{~cm}$ away from the midline.

The complex then excised full thickness to end of the sacral fascia with a straight edge on the side of flap mobilisation and a smooth edge on the other side. For bleeding control electrocautery (The Valleylab ${ }^{\mathrm{TM}}$, Covidien, USA) and hemostatic powder (Aris$\mathrm{ta}^{\mathrm{TM}}$, Bard, USA/Oxicel Powder, Betatech Med, Istanbul, Turkey) -by applying and holding 2 minutes- used for flap side. If hemorrhagia from flap side continues secondary application of powder then electrocauterisation planned. This is followed by mobilisation of the flap across the midline. 1-0 polyglactin sutures was used to put together the sacral fascia in the midline to the $V$ junction of the flap. A hemovac drain was placed. A second layer of polyglactin sutures used to secure the flap. Skin closed using 2-0 nylon mattress 
sutures and a pressure dressing was administered. Wound inspection was done on $1^{\text {st }}$ postoperative day and patient was discharged. Drains were removed 5 to 10 days later. Sutures were, usually, removed on the $11^{\text {th }}$ or $12^{\text {th }}$ postoperative day. All patients evaluated in respect of perioperative complications.

\section{Results}

44 of the initial sample of patients included in this study. The study population comprised of 44 patients, including 11 female and 33 male patients. The sex distribution was significantly in favour of male gender.

The mean age in is 27.00 ( \pm 7.18 years). The mean wound size $(\mathrm{mm})$ was $30.10( \pm 5.50) \mathrm{mm}$. And the complete wound healing time (days) was $10.5( \pm 2.10)$ days. The mean time of surgery duration (minutes) is $30.10( \pm 10.50)$ minutes. The mean length of hospital stay (days) is $1.50( \pm 0.50)$ days.

Postoperative complications were not common overall. The most common post-operative complication reported as seroma collection. 2 patients developed fluid collection under the flap site which reduce spontaneously. No hemorrhagia has occurred. 1 patient presented with wound infection after discharge. No hematoma, bleeding occurred and no need for secondary powder application. No flap necrosis occurred, also wound dehiscence and early recurrence were not found.

\section{Discussion and Conclusion}

Pilonidal sinus disease (PD) is not a rare condition, it mostly affects young men. Even though many surgical techniques have been described for treating PD, controversies regarding the best surgical approach still stands [6]. The main approach for operative management for chronic or persistent PD is excision of the entire pilonidal sinus complex including epithelialized tracts, using methylene blue to visualise the sinus tracts down to the level of the sacrococcygeal fascia [6-10]. Optimal closure of the defect following excision is controversial. Primary closure can be chosen by using either midline or off-midline techniques, including karydakis flap reconstruction [6].

Ideally, the main aim of treatment for this disease should be optimal wound healing with low risk of recurrence, patient comfort, low morbidity and reduced hospital stay time with few woundmanagement problems [11].

The Karydakis flap procedure was first presented by George Karydakis [3] in 1973 and has frequently been used to treat sacrococcygeal PD. The KF described symmetric closure of the pilonidal wounds by avoiding placement of the wound in the midline at the depth of the natal cleft and also flattens the cleft, thereby reducing hair accumulation and mechanical irritation and decreasing recurrence [11].
Considering the low recurrence rates in recurrent cases, Karydakis flap should be considered first. Even if flap techniques are unpopular, Karydakis flap is a preferable method for patients who have concerns about cosmetics and recurrence, which has also been reported in other studies [12].

Beyond recurrence our results shows Karydakis technique as a safe option.

When considered the low recurrence rates, patient tolerability and cosmetic outcomes, Karydakis technique emerges a method that is preferred by physicians and patients.

\section{Bibliography}

1. Gavriilidis P and Bota E. "Limberg flap versus Karydakis flap for treating pilonidal sinus disease: a systematic review and meta-analysis". Canadian Journal of Surgery 62.2 (2019): 131-138.

2. Hodges RM. "Pilonidal sinus". Boston Medical and Surgical Journal 103 (1880): 485-486.

3. Karydakis GE. "New approach to the problem of pilonidal disease”. Lancet 2.7843 (1973): 1414-1415.

4. Karydakis GE. "Easy and successful treatment of pilonidal sinus after explanation of its causative process". ANZ Journal of Surgery 62.5 (1992): 385-389.

5. Armstrong JH and Barcia PJ. "Pilonidal sinus disease. The conservative approach". Archives of Surgery 129.9 (1994): 914917.

6. Alvandipour M., et al. "Comparison of Limberg Flap and Karydakis Flap Surgery for the Treatment of Patients with Pilonidal Sinus Disease: A Single-Blinded Parallel Randomized Study". Annals of Coloproctology 35.6 (2019): 313-318.

7. Khanna A and Rombeau JL. "Pilonidal disease". Clinics in Colon and Rectal Surgery 24.1 (2011): 46-53.

8. Humphries AE and Duncan JE. "Evaluation and management of pilonidal disease". Surgical Clinics of North America 90.1 (2010): 113-124.

9. Oncel M., et al. "Excision and marsupialization versus sinus excision for the treatment of limited chronic pilonidal disease: a prospective, randomized trial". Techniques in Coloproctology 6.3 (2002): 165-169.

10. Bascom J. "Pilonidal disease: long-term results of follicle removal". Diseases of the Colon and Rectum 26.12 (1983): 800807. 
11. Bali İ., et al. "Effectiveness of Limberg and Karydakis flap in recurrent pilonidal sinus disease". Clinics (Sao Paulo) 70.5 (2015): 350-355.

12. Erkent M., et al. "Comparison of Primary Midline Closure, Limberg Flap, and Karydakis Flap Techniques in Pilonidal Sinus Surgery”. Medical Science Monitor 24 (2018): 8959-8963.

\section{Assets from publication with us}

- Prompt Acknowledgement after receiving the article

- Thorough Double blinded peer review

- Rapid Publication

- Issue of Publication Certificate

- High visibility of your Published work

Website: www.actascientific.com/

Submit Article: www.actascientific.com/submission.php Email us: editor@actascientific.com

Contact us: +91 9182824667 